\title{
Compression and tension variably alter Osteoprotegerin expression via miR-3198 in periodontal ligament cells
}

Hiroyuki Kanzaki ${ }^{1,2^{*}}$ D, Satoshi Wada ${ }^{2}$, Yuuki Yamaguchi ${ }^{2}$, Yuta Katsumata ${ }^{2}$, Kanako Itohiya ${ }^{2}$, Sari Fukaya ${ }^{2}$, Yutaka Miyamoto ${ }^{2}$, Tsuyoshi Narimiya $^{2}$, Koji Noda $^{2}$ and Yoshiki Nakamura ${ }^{2}$

\begin{abstract}
Background: Osteoclasts play a critical role in bone resorption due to orthodontic tooth movement (OTM). In OTM, a force is exerted on the tooth, creating compression of the periodontal ligament (PDL) on one side of the tooth, and tension on the other side. In response to these mechanical stresses, the balance of receptor activator of nuclear-factor kappa-B ligand (RANKL) and osteoprotegerin (OPG) shifts to stimulate osteoclastogenesis. However, the mechanism of OPG expression in PDL cells under different mechanical stresses remains unclear. We hypothesized that compression and tension induce different microRNA (miRNA) expression profiles, which account for the difference in OPG expression in PDL cells.

To study miRNA expression profiles resulting from OTM, compression force $\left(2 \mathrm{~g} / \mathrm{cm}^{2}\right)$ or tension force $(15 \%$ elongation) was applied to immortalized human PDL (HPL) cells for $24 \mathrm{~h}$, and miRNA extracted. The miRNA expression in each sample was analyzed using a human miRNA microarray, and the changes of miRNA expression were confirmed by real-time RT-PCR. In addition, miR-3198 mimic and inhibitor were transfected into HPL cells, and OPG expression and production assessed.

Results: We found that certain miRNAs were expressed differentially under compression and tension. For instance, we observed that miR-572, $-663,-575,-3679-5 p$, UL70-3p, and -3198 were upregulated only by compression. Real-time RT-PCR confirmed that compression induced miR-3198 expression, but tension reduced it, in HPL cells. Consistent with previous reports, OPG expression was reduced by compression and induced by tension, though RANKL was induced by both compression and tension. OPG expression was upregulated by miR-3198 inhibitor, and was reduced by miR-3198 mimic, in HPL cells. We observed that miR-3198 inhibitor rescued the compressionmediated downregulation of OPG. On the other hand, miR-3198 mimic reduced OPG expression under tension. However, RANKL expression was not affected by miR-3198 inhibitor or mimic.
\end{abstract}

Conclusions: We conclude that miR-3198 is upregulated by compression and is downregulated by tension, suggesting that miR-3198 downregulates OPG expression in response to mechanical stress.

Keywords: MicroRNA, Osteoprotegerin, Orthodontic tooth movement, miR-3198, Mechanical stresses

\footnotetext{
* Correspondence: kanzaki-h@tsurumi-u.ac.jp

${ }^{1}$ Tohoku University Hospital, Maxillo-oral Disorders, Sendai, Japan

${ }^{2}$ Department of Orthodontics, School of Dental Medicine, Tsurumi University,

2-1-3 Tsurumi, Tsurumi-ku, Yokohama, Kanagawa pref 230-8501, Japan
}

(C) The Author(s). 2019 Open Access This article is distributed under the terms of the Creative Commons Attribution 4.0 International License (http://creativecommons.org/licenses/by/4.0/), which permits unrestricted use, distribution, and reproduction in any medium, provided you give appropriate credit to the original author(s) and the source, provide a link to the Creative Commons license, and indicate if changes were made. The Creative Commons Public Domain Dedication waiver (http://creativecommons.org/publicdomain/zero/1.0/) applies to the data made available in this article, unless otherwise stated. 


\section{Background}

Orthodontic tooth movement (OTM) describes the orchestrated responses of periodontal tissues in response to physical force. During OTM, site-specific bone metabolisms take place simultaneously: osteoclastic bone resorption in the compression zone and osteoblastic bone formation in the tension zone of periodontal ligament (PDL) [1-3]. Osteoclastogenesis is primarily regulated by receptor activator of nuclearfactor kappa-B ligand (RANKL) [4]. RANKL signaling is inhibited by osteoprotegerin (OPG), and the balance between RANKL and OPG contributes to the regulation of bone resorption [5]. The relationship between the RANKL/OPG ratio and progression of OTM has been extensively studied. Compression induces RANKL expression [6-9] and reduces OPG expression $[10,11]$ in PDL cells, thereby increasing the RANKL/OPG ratio, and favoring RANKL-mediated osteoclastogenesis. Conversely, tension increases OPG expression in PDL cells both in vivo [12,13] and in vitro [14-17], which in turn inhibits RANKL. However, the mechanism regulating OPG expression in PDL cells under different mechanical stresses remains unclear.

The relationship between mechano-sensing and microRNA (miRNA) expression has recently become clearer. It is now understood that miRNAs in vascular endothelial cells play an essential role in shear stress-regulated endothelial responses [18]. Mechanical stretch regulates microRNA expression in C2C12 myoblasts [19]. Furthermore, mechanical stress can induce expression of miRNAs that modulate the expression of osteogenic and bone resorption factors, thus effecting bone remodeling due to mechanical stresses [20]. These studies suggest miRNAs might play a role in the regulation of differential OPG expression in PDL cells under compression and tension.

We hypothesized that compression and tension induce different miRNA expression profiles, resulting in differential OPG expression in PDL cells. To test this hypothesis, in the present study we used miRNA microarrays to examine miRNA expression profiles of cultured PDL cells under compression and tension. We identified several miRNAs that were differentially regulated during compression and tension, and, using target prediction databases, identified OPG as a potential target for the miRNA miR-3198. We found miR-3198 was upregulated by compression and downregulated by tension. Augmentation and attenuation of miR-3198 by a miRNA mimic and an inhibitor, respectively, revealed that $O P G$ expression was downregulated by $m i R-3198$.

Thus, we show here that compression and tension differentially regulate miRNA expression. Importantly, we found that miR-3198, which was induced by compression and reduced by tension, downregulates OPG expression in PDL cells.

\section{Results \\ miRNA expression is mediated by mechanical stress}

We examined miRNA expression in human PDL (HPL) cells in three different groups (control, compression, and tension) using a microarray. The top 20 differentially expressed miRNAs between the control and compression groups, the control and tension groups, and the tension and compression groups were identified (Table 1). Some miRNAs, such as miR-1268, - 3648, $-642 b$, and $-135 a$, were upregulated in both the compression and tension groups compared with the control group. The data suggest these miRNAs are upregulated by any type of mechanical stress. miRNAs such as miR-572, - 663, - 575, - 3679-5p, UL70-3p, and - 3198 were upregulated in the compression group compared with both the control group and tension group, suggesting that these miRNAs are specifically upregulated by compression. miRNAs upregulated in the tension group relative to the control group included miR-376a. These results suggest that some miRNAs are upregulated in response to any type of mechanical stress, whilst other miRNAs are upregulated only in response to a specific type of mechanical stress.

miRNAs targeting OPG are regulated by mechanical stress Since OPG expression is regulated by mechanical stress in OTM, we investigated whether miRNAs upregulated by mechanical stress were also miRNAs predicted to target OPG. miRNAs that target OPG were predicted by two databases (Table 2). miR-1207 was on neither list; therefore an miR-1207 mimic and inhibitor were used as negative controls. We identified miR-3198 on both of the lists, raising the possibility that compression-induced miR-3198 downregulates OPG expression.

\section{Expression of miR-3198 and OPG was regulated differentially in compression and tension}

Real-time RT-PCR analysis confirmed the effects of 3198 due to mechanical stress. Compression induced miR-3198 expression in HPL cells, whereas tension reduced miR-3198 expression (Fig. 1a). These results were consistent with the miRNA array analysis. OPG expression was reduced by compression but was induced by tension (Fig. 1b). RANKL expression was increased by both compression and tension (Fig. 1c). Western blotting revealed that compression reduced OPG protein level in the cultured HPL cells (Fig. 1d and e). In addition, tension increased OPG protein level in the cultured HPL cells. These results suggest that miR-3198 downregulates OPG expression in response to mechanical stress. 
Table 1 microarray analysis for miRNA expression

\begin{tabular}{|c|c|c|c|c|c|}
\hline control VS Compression & Log2 Ratio & control VS Tension & Log2 Ratio & Tension VS Compression & Log2 Ratio \\
\hline hsa-miR-1268 & 9.94 & hsa-miR-3648 & 7.37 & hsa-miR-4299 & 11.10 \\
\hline hsa-miR-572 & 7.73 & hsa-miR-1268 & 6.57 & hsa-miR-572 & 7.73 \\
\hline hsa-miR-663 & 7.60 & hsa-miR-642b & 6.31 & hsa-miR-663 & 7.60 \\
\hline hsa-miR-3648 & 7.21 & hsa-miR-135a & 5.35 & hsa-miR-575 & 6.87 \\
\hline hsa-miR-575 & 6.87 & hsa-miR-376a & 5.21 & hsa-miR-3679-5p & 6.68 \\
\hline hsa-miR-3679-5p & 6.68 & hsa-miR-4271 & 1.64 & hemv-miR-UL70-3p & 6.57 \\
\hline hsa-miR-642b & 6.64 & hsa-miR-136 & 1.47 & hsa-miR-3198 & 6.56 \\
\hline hemv-miR-UL70-3p & 6.57 & hsa-miR-29b & 1.36 & hsa-miR-1305 & 6.47 \\
\hline hsa-miR-3198 & 6.56 & hsa-miR-3663-3p & 1.36 & hsa-miR-1225-3p & 6.31 \\
\hline hsa-miR-1305 & 6.47 & hsv1-miR-H18 & 1.28 & hsa-miR-125a-3p & 6.16 \\
\hline hsa-miR-1225-3p & 6.31 & hsa-miR-3656 & -1.04 & hsa-miR-1246 & 6.14 \\
\hline hsa-miR-125a-3p & 6.16 & ebv-miR-BART13 & -1.16 & hsv1-miR-H17 & 5.88 \\
\hline hsa-miR-1246 & 6.14 & hsa-miR-145 & -1.28 & hsa-miR-140-3p & 5.79 \\
\hline hsv1-miR-H17 & 5.88 & hsa-miR-181b & -1.35 & hsa-miR-155 & 5.75 \\
\hline hsa-miR-654-5p & 5.48 & hsa-miR-181a-2 & -1.42 & hsa-miR-654-5p & 5.48 \\
\hline hsa-miR-135a & 5.43 & hsa-miR-503 & -4.99 & hsa-miR-129-3p & 5.37 \\
\hline hsa-miR-129-3p & 5.37 & hsa-miR-425 & -5.43 & hsa-miR-874 & 5.36 \\
\hline hsa-miR-874 & 5.36 & hsa-miR-425 & -5.61 & hsa-miR-425 & 5.26 \\
\hline hsa-miR-4299 & 5.32 & hsa-miR-4299 & -5.78 & hsv1-miR-H7 & 5.26 \\
\hline hsv1-miR-H7 & 5.26 & hsa-miR-155 & -5.87 & hsa-miR-485-3p & 5.26 \\
\hline
\end{tabular}

miR-3198 gain-of-function and loss-of-function experiments

We further examined the relationship between miR-3198 and OPG expressions through gain- and loss-of-function experiments. We found that transfection of miR-3198 inhibitor in HPL cells reduced miR-3198 expression (Fig. 2a), whereas transfection of miR-3198 mimic upregulated miR-3198 expression (Fig. 2b). Similarly, OPG mRNA expression was induced by miR-3198 inhibitor (Fig. 2c) and reduced by miR-3198 mimic (Fig. 2d). RANKL mRNA expression was stable irrespective of the transfection of miR-3198 inhibitor or mimic. OPG protein levels were also increased by miR-3198 inhibitor (Fig. 2e) and decreased by miR-3198 mimic (Fig. 2f).

To clarify whether these phenomena were dependent on miR-3198 specifically, we examined the role of miR-1207, which was not predicted to target OPG. We found that the transfection of miR-1207 inhibitor reduced miR-1207 expression in HPL cells (Fig. 2g), and the transfection of miR-1207 mimic upregulated miR-1207 expression (Fig. $2 \mathrm{~h}$ ), consistent with the results of the miR-3198 inhibitor and mimic experiment. OPG and RANKL expression were stable regardless of the transfection of miR-1207 inhibitor or mimic (Fig. 2i and $j$ ).

These results suggest that mechanical stress-induced miR-3198 downregulates OPG expression in HPL cells.

\section{miR-3198 regulates mechanical stress-mediated OPG expression}

Finally, we examined the role of miR-3198 in the regulation of mechanical stress-mediated OPG expression. We found that OPG expression was reduced by compression, although transfection of miR-3198 inhibitor rescued the compression-mediated downregulation of OPG (Fig. 3a). In addition, there was no significant difference between the control and the compression + miR-3198 inhibitor groups, indicating that miR-3198 plays a role in the regulation of OPG expression under compression. RANKL mRNA expression was upregulated by compression, and it was stable irrespective of the transfection of miR-3198 inhibitor.

Conversely, we found that under tension, augmentation of miR-3198 expression by miR-3198 mimic reduced $O P G$ expression (Fig. 3b). There was a significant difference in the OPG expression levels between the control and the tension + miR-3198 mimic $(P=0.03)$. $R A N K L$ mRNA expression was upregulated by tension, and it was stable irrespective of the transfection of miR-3198 mimic.

Consistent with results of real-time PCR, OPG quantification by ELISA revealed that compression reduced OPG protein levels (Fig. 3c), whereas miR-3198 inhibitor prevented the compression-mediated reduction of OPG. In addition, tension upregulated OPG production (Fig. 
Table 2 microRNAs which target OPG (TNFRSF11b)

\begin{tabular}{|c|c|c|c|c|c|c|c|}
\hline \multicolumn{4}{|c|}{ microRNA.org } & \multicolumn{4}{|c|}{ miRDB.org } \\
\hline \multicolumn{4}{|c|}{ RANK and miRNA } & \multicolumn{4}{|c|}{ RANK and miRNA } \\
\hline 1 & hsa-miR-3163 & 26 & hsa-miR-135a & 1 & hsa-let-7f-2-3p & 26 & hsa-miR-6870-3p \\
\hline 2 & hsa-miR-586 & 27 & hsa-miR-135b & 2 & hsa-miR-1185-1-3p & 27 & hsa-miR-936 \\
\hline 3 & hsa-miR-633 & 28 & hsa-miR-200b & 3 & hsa-miR-1185-2-3p & 28 & hsa-miR-5692a \\
\hline 4 & hsa-miR-656 & 29 & hsa-miR-590-5p & 4 & hsa-miR-4262 & 29 & hsa-miR-145-5p \\
\hline 5 & hsa-miR-130b & 30 & hsa-miR-21 & 5 & hsa-miR-3163 & 30 & hsa-miR-5195-3p \\
\hline 6 & hsa-miR-548c-3p & 31 & hsa-miR-4255 & 6 & hsa-miR-892c-5p & 31 & hsa-let-7c-3p \\
\hline 7 & hsa-miR-590-3p & 32 & hsa-miR-4309 & 7 & hsa-miR-5584-5p & 32 & hsa-miR-216a-5p \\
\hline 8 & hsa-miR-577 & 33 & hsa-miR-3198 & 8 & hsa-miR-4729 & 33 & hsa-miR-4753-3p \\
\hline 9 & hsa-miR-579 & 34 & hsa-miR-2054 & 9 & hsa-miR-181a-5p & 34 & hsa-miR-590-5p \\
\hline 10 & hsa-miR-576-5p & 35 & hsa-miR-936 & 10 & hsa-miR-181c-5p & 35 & hsa-miR-3160-5p \\
\hline 11 & hsa-miR-429 & 36 & hsa-miR-380 & 11 & hsa-miR-181d-5p & 36 & hsa-miR-429 \\
\hline 12 & hsa-miR-488 & 37 & hsa-miR-3172 & 12 & hsa-miR-181b-5p & 37 & hsa-miR-200b-3p \\
\hline 13 & hsa-miR-4262 & 38 & hsa-miR-376a & 13 & hsa-miR-3942-3p & 38 & hsa-miR-200c-3p \\
\hline 14 & hsa-miR-181a & 39 & hsa-miR-376b & 14 & hsa-miR-4766-3p & 39 & hsa-miR-765 \\
\hline 15 & hsa-miR-181b & 40 & hsa-miR-145 & 15 & hsa-miR-4668-5p & 40 & hsa-miR-5582-5p \\
\hline 16 & hsa-miR-181c & 41 & hsa-miR-193b & 16 & hsa-miR-3942-5p & 41 & hsa-miR-629-5p \\
\hline 17 & hsa-miR-181d & 42 & hsa-miR-4307 & 17 & hsa-miR-4294 & 42 & hsa-miR-6892-5p \\
\hline 18 & hsa-miR-1283 & 43 & hsa-miR-765 & 18 & hsa-miR-4703-5p & 43 & hsa-miR-577 \\
\hline 19 & hsa-let-7f-2 & 44 & hsa-miR-374a & 19 & hsa-miR-6501-3p & 44 & hsa-miR-579-3p \\
\hline 20 & hsa-miR-889 & 45 & hsa-miR-29b-2 & 20 & hsa-miR-506-3p & 45 & hsa-miR-183-3p \\
\hline 21 & hsa-miR-4294 & 46 & hsa-miR-570 & 21 & hsa-miR-124-3p & 46 & hsa-miR-6074 \\
\hline 22 & hsa-miR-187 & 47 & hsa-miR-188-3p & 22 & hsa-miR-3662 & 47 & hsa-miR-3198 \\
\hline 23 & hsa-miR-200c & 48 & hsa-miR-222 & 23 & hsa-miR-130b-5p & 48 & hsa-miR-513b-3p \\
\hline 24 & hsa-miR-506 & 49 & hsa-miR-3170 & 24 & hsa-miR-193b-5p & 49 & hsa-miR-7109-3p \\
\hline 25 & hsa-miR-124 & 50 & hsa-miR-1323 & 25 & hsa-miR-5582-3p & 50 & hsa-miR-4309 \\
\hline
\end{tabular}

miR-3198, which is found in the table 1 , is indicated by boldface

3d). Similarly, we found that miR-3198 mimic significantly reduced the tension-mediated increase in OPG production.

Western blotting for OPG also revealed that the miR-3198 inhibitor prevented the compression-mediated reduction of OPG (Fig. 3e and g). On the other hand, miR-3198 mimic significantly reduced the tension -mediated increase in OPG production (Fig. $3 \mathrm{f}$ and $\mathrm{h}$ ). These results indicate that miR-3198 downregulates OPG expression in HPL cells under mechanical stress.

\section{Discussion}

Osteoclastic bone resorption is tightly regulated by RANKL [4] in the periodontal ligament during OTM [7, 10]. Conversely, OPG, the decoy receptor to RANKL, inhibits osteoclastogenesis [21]. The RANKL/OPG ratio increases at the compression zone of the PDL during OTM [10]. In vitro experiments have revealed that compression increases the RANKL/OPG ratio in PDL cells [9, 22-24]. On the other hand, tension decreases
RANKL/OPG ratio in PDL cells, mainly by the induction of OPG expression [14-17]. Generally, OPG expression in the PDL cells usually increases under tension and decreases under compression. Though our previous report [15] and this study revealed upregulation of RANKL by tension, induction of OPG by tension predominates over RANKL, resulting in a low RANKL/OPG ratio and inactive osteoclastogenesis.

In this study, we examined whether miRNAs are involved in site-specific changes in OPG expression during OTM. We found that miRNAs were differentially regulated by compression and tension. In particular, miR-3198 was upregulated by compression and downregulated by tension. Furthermore, we found that miR-3198 regulates OPG expression in response to mechanical stresses, which is consistent with phenomena observed in the PDL during OTM; namely, osteoclastic bone resorption in the compression zone and osteoblastic bone formation in the tension zone of the PDL [3]. On the other hand, RANKL expression was not affected by miR-3198. 


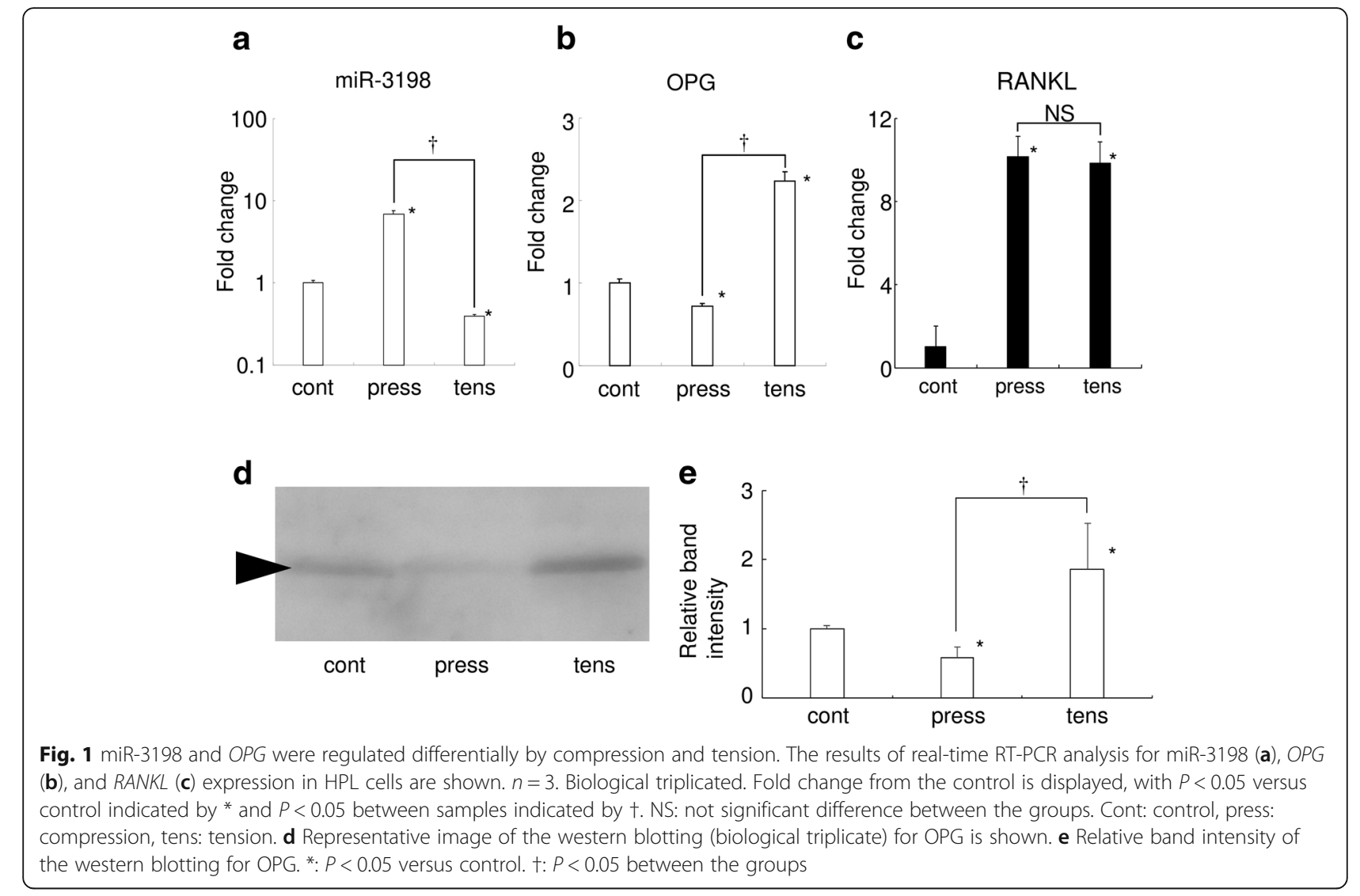

OPG

C

Our present results indicate that tension-induced OPG expression is reduced by the overexpression of miR-3198 mimic, although we did find a significant difference in OPG expression between control and tension + miR-3198 mimic groups. Considering that miR-3198 plays a role in the regulation of OPG expression under different mechanical stresses, epigenetic regulation such as methylation of miR-3198 and mutation of miR-3198 would interfere the mechanical stress-mediated miR-3198 expression followed by the difference in OPG expression, which regulates the alveolar bone resorption during orthodontic tooth movement. Further studies will shed light on the importance of miR-3198 on the regulation of the alveolar bone metabolism during orthodontic tooth movement.

Regarding the relationship between OTM and miRNA, Chen et al. reported that miR-21 deficiency attenuated OTM via inhibition of alveolar bone resorption on both the compressive and tensile sides [25]. In addition, Chang et al. reported the role of miRNA in tension force-induced bone formation [26]. They concluded that miR-195-5p, miR-424-5p, miR-1297, miR-3607-5p, miR-145-5p, miR-4328, and miR-224-5p were core miRNAs of tension force-induced bone formation. Within these miRNA, no miRNAs were changed by mechanical stresses in our experiment, except miR-145. We presumed that the difference in observed miRNAs between Chang's study and ours would be due to the different time points assessed (at $72 \mathrm{~h}$ in Chang's paper, and at $24 \mathrm{~h}$ in ours). We wanted to explore the early response of PDL cells against mechanical stress via miRNA, and examined only at $24 \mathrm{~h}$, the timing the other researchers tested at [27, 28]. Exploration of the time course change of each miRNA would be useful to clarify this. Liu et al. reported that miR-503-5p functions as a mechano-sensitive miRNA and inhibits bone marrow stromal cell osteogenic differentiation subjected to mechanical stretch and bone formation in OTM tension sides [29]. Chen et al. reported that cyclic stretch decreased, and compression increased, the expression of miR-29 in PDL cells, which directly interacts with Col1a1, Col3a1 and Col5a1 [30]. These studies reveal the relationship between mechanical stress-mediated miRNA expression and bone formation or tissue remodeling. However, the effects of miRNA on the RANKL/ OPG ratio during OTM were unclear until now.

Regarding the regulation of OPG expression by miRNAs, miRs-21 [31, 32], - 145 [33], -146a [34], - 150 [35], and - 200 [36] have been reported to regulate OPG 
a

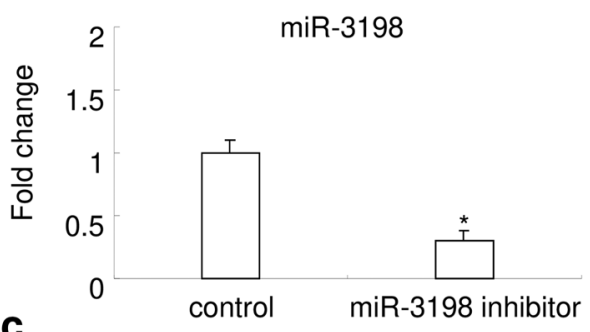

b
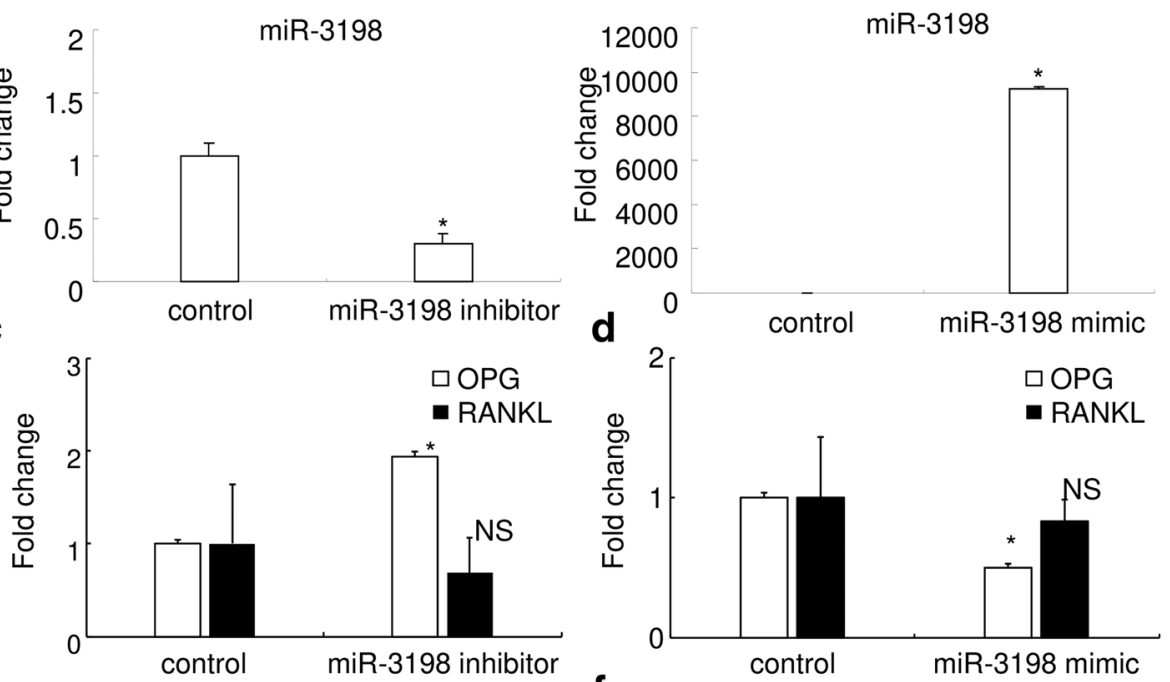

e
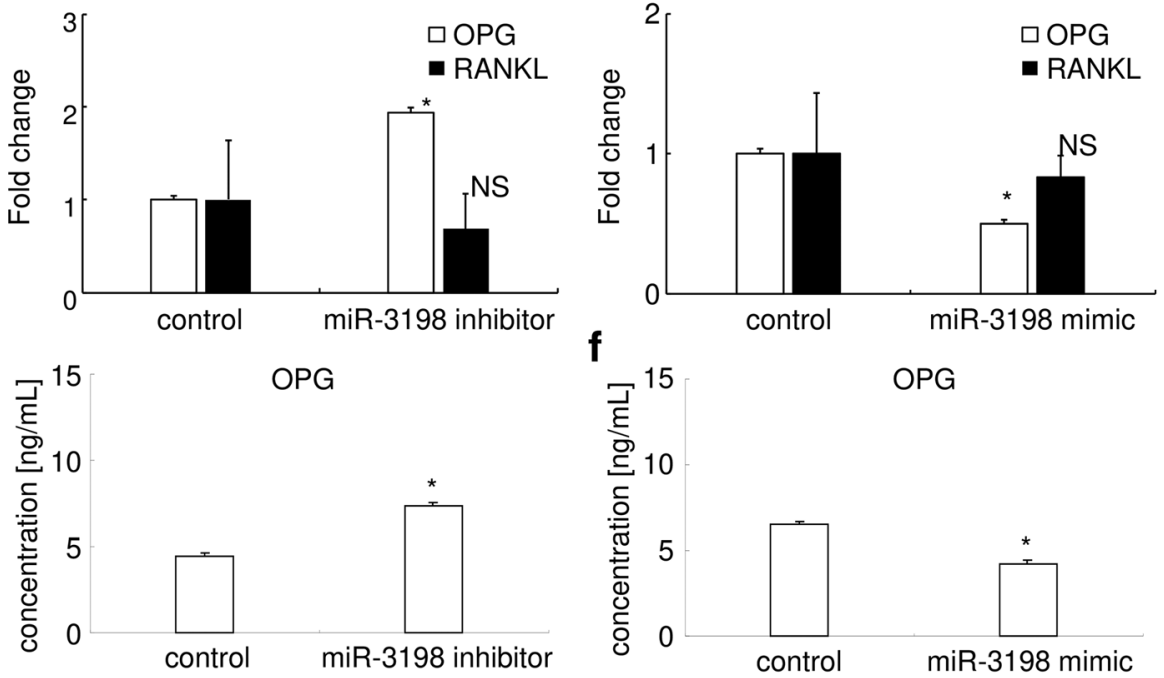

$f$

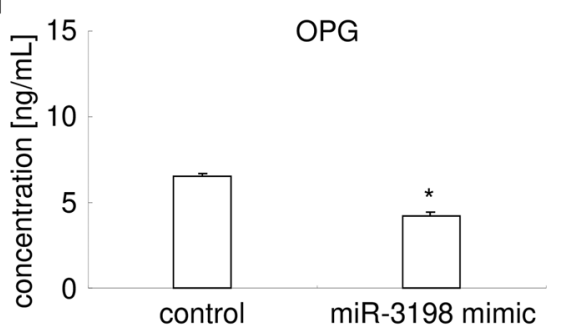

g

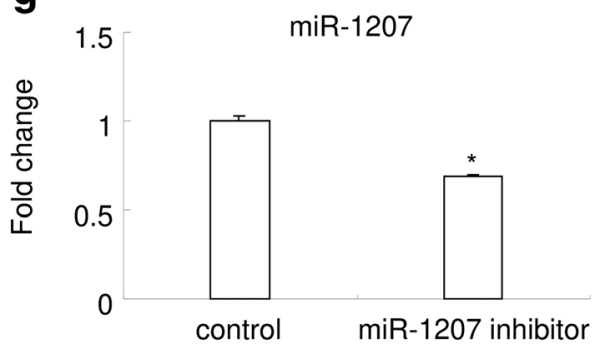

h

8000

miR-1207

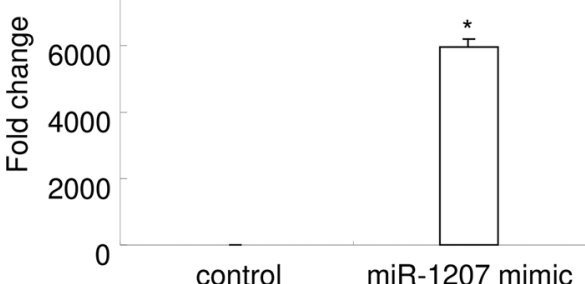

i

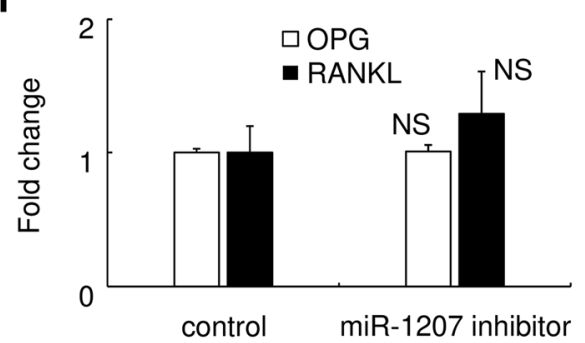

j

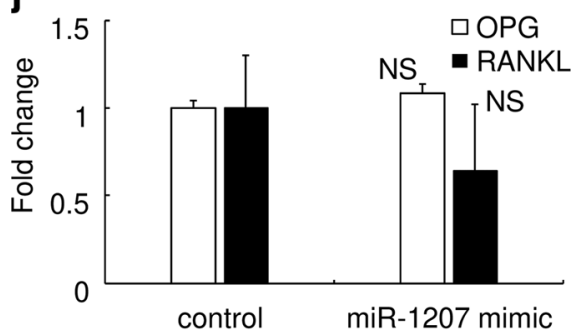

Fig. 2 miR-3198 gain-of-function and loss-of-function experiments. Results of real-time RT-PCR analysis for miR-3198 (a, b), OPG and RANKL (c, d) expression in HPL cells after transfection of miR-3198 inhibitor $(\mathbf{a}, \mathbf{c})$ and miR-3198 mimic $(\mathbf{b}, \mathbf{d}) . n=3$. Biological triplicated. Fold change from the control is shown. Open bar indicates the fold change of OPG expression, and close bar indicates that of RANKL expression (c, d). The concentrations of OPG as measured by ELISA after transfection of miR-3198 inhibitor $(\mathbf{e})$ and miR-3198 mimic $(\mathbf{f})$ are shown $(n=3)$. Results of real-time PCR analysis for miR-1207 ( $\mathbf{g}, \mathbf{h})$, OPG and RANKL (i, j) expression in HPL cells under the transfection of miR-1207 inhibitor $(\mathbf{g}, \mathbf{i})$ and miR-1207 mimic $(\mathbf{h}, \mathbf{j}) . n=3$. Biological triplicated. Fold changes from the control are shown. ${ }^{*}$ indicates $P<0.05$ versus control, and NS indicating there was no significant difference between samples 


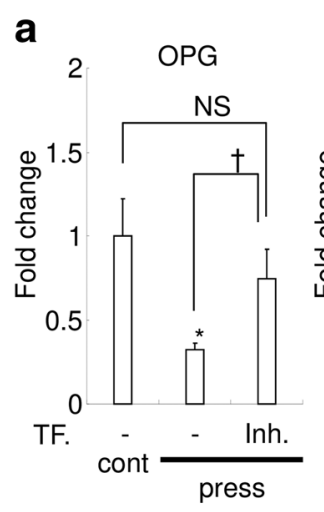

C

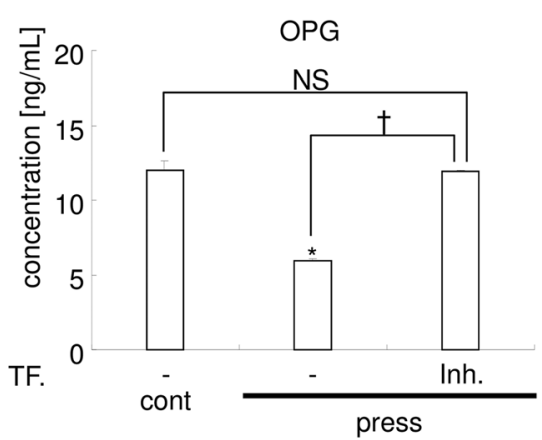

e

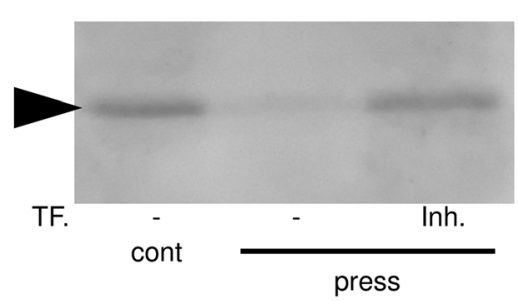

g

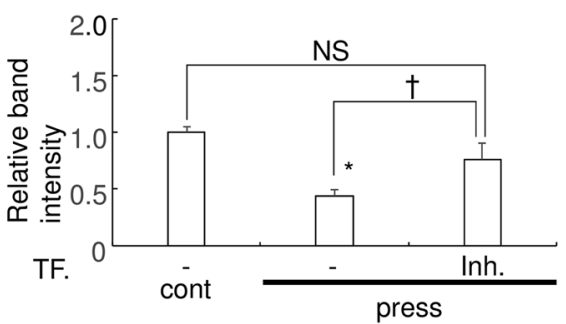

b $\quad O P G$

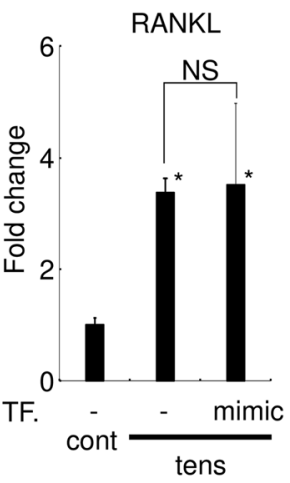

d

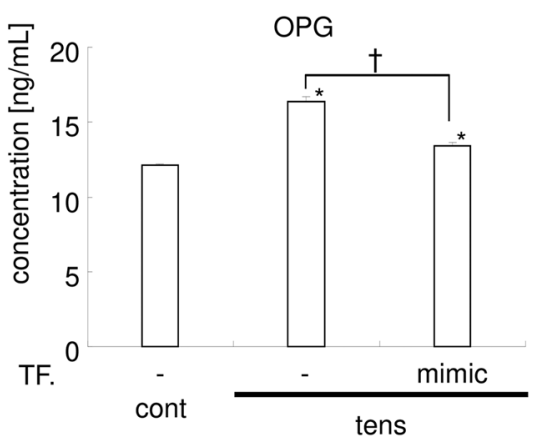

f

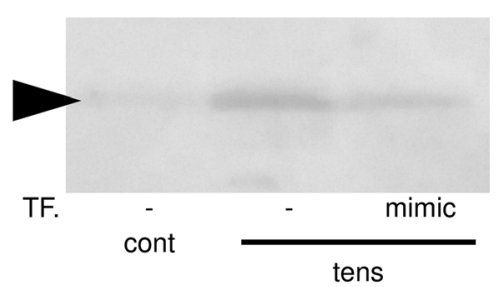

h

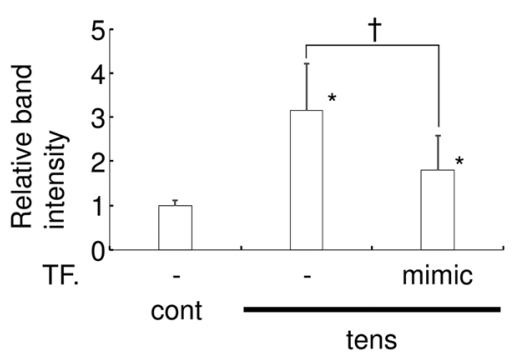

Fig. 3 miR-3198 regulates the mechanical stress-mediated change of OPG expression. Results of real-time RT-PCR analysis for OPG and RANKL expression in HPL cells in the compression (a) and tension (b) experiments. $n=3$. Biological triplicated. Fold change from the control are shown. Cont, control; Inh, transfection of miR-3198 inhibitor; Mimic, transfection of miR-3198 mimic; press, compression; tens, tension; TF, transfection. Also shown are the OPG concentrations measured by ELISA in the compression $(\mathbf{c})$ and tension $(\mathbf{d})$ experiments $(n=3)$. ${ }^{*}$ indicates $P<0.05$ versus control. + indicates $P<0.05$ between samples. NS indicates there was no significant difference between samples. e and $\mathbf{f}$ Representative image of the western blotting for OPG was shown. $\mathbf{g}$ and $\mathbf{h}$ Relative band intensity of the western blotting for OPG. * $P<0.05$ versus control. t: $P<0.05$ between the groups. NS, no significant difference between the samples

expression. Among them, miRs-21, -145 , and -200 were thought to be direct regulators of OPG expression in the databases of miRDB.org and microRNA.org. Therefore, we presumed that the refinement of candidate miRNAs using the available databases was a sufficiently accurate method to choose candidates.
We found that miR-3198 plays a role in the regulation of the mechanical stress-mediated OPG expression, although the reciprocal regulatory mechanism of miR-3198 by compression and tension remains unclear. Some mechanical stresses induce differential intracellular signaling systems, such as G-proteins, calcium 
signaling, MAPK signaling, and nitric oxide signaling [37]. Further studies are needed to clarify the regulatory mechanism of miR-3198 by compression and tension. miR-3198 was identified in human tumor breast tissue [38], and is on 22q11.21 of the genome. It is important to confirm that miR-3198 downregulates OPG expression by mechanical stress in animal models. However, there is no orthologue of miR-3198 in mice or rats, which makes it difficult to conduct such experiments. Nevertheless, further confirmatory experiments are required.

\section{Conclusions}

In conclusion, we found that miRNAs were differentially regulated by compression and tension in PDL cells. Furthermore, miR-3198 downregulates OPG expression in PDL cells in response to mechanical stress.

\section{Methods \\ Cells}

Human immortalized periodontal ligament cell lines (HPL cells) were received from the University of Hiroshima, Hiroshima, Japan, where they were originally established [39]. HPL cells were cultured in alpha modified Eagle's medium (Wako Pure Chemical, Osaka, Japan) containing $10 \%$ fetal bovine serum (Thermo Fisher Scientific, Waltham, MA) and supplemented with penicillin $(100 \mathrm{U} / \mathrm{mL})$ and streptomycin $(100 \mu \mathrm{g} / \mathrm{mL})$. All cells were cultured at $37^{\circ} \mathrm{C}$ in a $5 \% \mathrm{CO}_{2}$ incubator.

\section{Application of mechanical stress}

Compressive force was applied to the HPL cells using a glass cylinder, as described elsewhere [9]. Briefly, a glass cylinder was placed over a confluent cell layer in the well of a 6-well plate. HPL cells were subjected to $2 \mathrm{~g} / \mathrm{cm}^{2}$ of compressive force for $24 \mathrm{~h}$. Cyclical tensile force was applied to HPL cells with a Flexercell Strain-Unit (Flexcell Corp., Hillsborough, NC, USA), as described elsewhere [15]. Briefly, PDL cells were pre-cultured in flexiblebottomed culture plates coated with type I collagen until confluent. The culture plates were then set on the rubber gasket of the Flexercell Strain Unit, and PDL cells were subjected to cyclical tensile force (15\% elongation, $1 \mathrm{~s}$ stretch $/ 1 \mathrm{~s}$ relaxation) for $24 \mathrm{~h}$.

\section{miRNA and RNA extraction}

miRNA and RNA were extracted separately from HPL cells using the Nucleospin miRNA isolation kit (Macherey-Nagel, Düren, Germany), according to the manufacturer's instructions.

\section{miRNA array analysis}

The quality of the extracted miRNAs was examined by an Agilent 2100 Bioanalyser (Agilent Technologies,
Santa Clara, CA). RNA integrity numbers ranged from 8.7 to 9.5. miRNA expression in each sample was analyzed using a SurePrint G3 Human miRNA microarray $8 \times 60 \mathrm{~K}$ miRBase 16.0 (Agilent Technologies), according to the manufacturer's instructions.

\section{Database analysis for miRNAs target prediction}

To identify candidate miRNA which targeted OPG, two target prediction databases, miRDB.org [40] and microRNA.org [41], were used. Candidate miRNAs were queried using "OPG" or "TNFRSF11B" as keywords.

\section{Reverse transcription (RT) and real-time RT-PCR analysis}

Isolated miRNA ( $2 \mu \mathrm{g}$ each) were reverse-transcribed (RT) with the miScript II RT kit (Qiagen, Germantown, MD), according to the manufacturer's instructions. After reverse transcription, cDNA samples were diluted $5 \times$ with TE buffer. Real-time RT-PCR was performed using the miScript SYBR green PCR kit (Qiagen). The following PCR primers were used for the detection of miRNA: miR-1207 (MIMAT0005871), miR-3198 (MIMAT0015083), and $R N U 6 B$. Fold change of miR-3198 expression relative to the control was calculated by the $\Delta-\Delta$ Ct method with $R N U 6 B$ as a reference gene. Isolated RNA (500 ng) was reverse-transcribed using the iScript cDNA-Supermix (Bio-Rad, Hercules, CA, USA), according to the manufacturer's instruction. After reverse transcription, cDNA samples were diluted $5 \times$ with TE buffer. Real-time RT-PCR was performed using the SsoFast EvaGreen-Supermix (Bio-Rad). PCR primers used for the experiments were human OPG (forward, 5' -AAGGGCGCTACCTTGAGAT AG-3'; reverse, 5'-GCAAACTGTATTTCGCTCTGGG -3'), human RANKL (forward, 5' -CGTTGGATCACAGC ACATCAG-3'; reverse, 5'-GCTCCTCTTGGCCAGAT CTAAC-3'), and human ribosomal protein S18 (RPS18) (forward, 5'-GATGGGCGGCGGAAAATAG-3'; reverse, 5'-GCGTGGATTCTGCATAATGGT-3'). Fold changes of $O P G$ and $R A N K L$ expression relative to the control were calculated by the $\Delta-\Delta$ Ct method with $R P S 18$ as a reference gene.

\section{miR-3198 gain-of-function and loss-of-function experiments}

To observe the influence of miR-3198 on OPG expression, miR-3198 mimic (Qiagen) and miR-3198 inhibitor (Qiagen) were transfected into HPL cells using the TransIT-TKO ${ }^{\circ}$ transfection reagent (Mirus Bio LLC, Madison, WI), according to the manufacturer's instructions. miR-1207 mimic (Qiagen) and miR-1207 inhibitor (Qiagen) were used as the negative control. miR mimic and $\mathrm{miR}$ inhibitor were used at a final concentration of $50 \mathrm{nM}$ (stock concentration, $20 \mu \mathrm{M}$ ), according to the manufacturer's recommendation. The expression of miR-3198 was observed at $24 \mathrm{~h}$ after transfection. In 
some experiments, mechanical stress was applied to transfected HPL cells, beginning $12 \mathrm{~h}$ after transfection.

\section{OPG ELISA}

The concentration of OPG in the culture supernatant was measured using an OPG ELISA kit (Boster Biological Technology, Pleasanton, CA), according to the manufacturer's instructions. Culture supernatants were diluted $5 \times$ prior to measurement.

\section{Western blotting for OPG}

Culture supernatants were subjected to electrophoresis on TGX Precast gels (BioRad), proteins were transferred to a PVDF membrane, which was blocked with PVDF Blocking Reagent (Toyobo Co. Ltd., Osaka, Japan), then incubated with a rabbit IgG anti-OPG antibody (GeneTex, Irvine, CA, USA). After thorough washing with $0.5 \%$ Tween-20 in PBS (PBS-T), the membrane was incubated with a horseradish peroxidase-conjugated anti-rabbit IgG antibody (R\&D Systems, Inc., Minneapolis, MN, USA). Chemiluminescence was produced by using Luminata-Forte (EMD Millipore, Billerica, MA) and detected with LumiCube (Liponics, Tokyo, Japan).

\section{Statistical analysis}

All data are presented as mean \pm SD. Comparisons between two groups were performed using Student's t-test. Multiple comparisons were performed by using Tukey's test. A $P$-value $<0.05$ was considered statistically significant.

\section{Abbreviations \\ ELISA: Enzyme-linked immunoSorbent assay; HPL cells: Human immortalized periodontal ligament cell lines; miRNA: MicroRNA; OPG: Osteoprotegerin; OTM: Orthodontic tooth movement; PDL: Periodontal ligament; RANKL: Receptor activator of NF-KB ligand; RT-PCR: Reverse transcription polymerase chain reaction; TGF-beta: Transforming growth factor beta}

\section{Acknowledgements}

We acknowledge Drs. Takashi Takata and Masae Kitagawa (University of Hiroshima, Hiroshima, Japan) for providing human immortalized periodontal ligament cell lines. We also acknowledge the Division of Oral Physiology, Tohoku University Graduate School of Dentistry, for their generous permission to use the Flexercell Strain-Unit. The authors acknowledge the Center of Research Instruments, Institute of Development, Aging and Cancer, Tohoku University, for generous permission to use the experimental instruments. Finally, the authors give their heartfelt appreciation to the experimental reagent companies and instrument companies for their various forms of support in the rehabilitation from the damage caused by the Tohoku earthquake on March 11, 2011.

\section{Funding}

This work was supported by Grants-in-Aid for Scientific Research from the Japan Society for the Promotion of Science (JP23689081, JP25670841, JP15K11356, JP16H05552, and JP15K11376).

\section{Availability of data and materials}

The datasets used and/or analysed during the current study are available from the corresponding author on reasonable request.

\section{Authors' contributions}

$H K, K N$, and $Y N$ made substantial contributions to conception and design. SW, YY, YK, KI, SF, YM, and TN made substantial acquisition of data, or analysis and interpretation of data. HK and YN wrote and edited the paper. All authors read and approved the final manuscript.

Ethics approval and consent to participate

Not applicable

\section{Consent for publication}

Not applicable

\section{Competing interests}

The authors declare that they have no competing interests.

\section{Publisher's Note}

Springer Nature remains neutral with regard to jurisdictional claims in published maps and institutional affiliations.

Received: 23 July 2018 Accepted: 19 March 2019

Published online: 04 April 2019

\section{References}

1. Brudvik P, Rygh P. Multi-nucleated cells remove the main hyalinized tissue and start resorption of adjacent root surfaces. Eur J Orthod. 1994;16(4):265-73.

2. Davidovitch Z, Nicolay OF, Ngan PW, Shanfeld JL. Neurotransmitters, cytokines, and the control of alveolar bone remodeling in orthodontics. Dent Clin N Am. 1988:32(3):411-35.

3. Storey E. The nature of tooth movement. Am J Orthod. 1973;63(3):292-314.

4. Udagawa N, Takahashi N, Jimi E, Matsuzaki K, Tsurukai T, Itoh K, Nakagawa N, Yasuda H, Goto M, Tsuda E, et al. Osteoblasts/stromal cells stimulate osteoclast activation through expression of osteoclast differentiation factor/ RANKL but not macrophage colony-stimulating factor: receptor activator of NF-kappa B ligand. Bone. 1999;25(5):517-23.

5. Hofbauer LC, Khosla S, Dunstan CR, Lacey DL, Boyle WJ, Riggs BL. The roles of osteoprotegerin and osteoprotegerin ligand in the paracrine regulation of bone resorption. J Bone Miner Res. 2000;15(1):2-12.

6. Sasaki T. Differentiation and functions of osteoclasts and odontoclasts in mineralized tissue resorption. Microsc Res Tech. 2003;61(6):483-95.

7. Yamaguchi M. RANK/RANKL/OPG during orthodontic tooth movement. Orthod Craniofac Res. 2009;12(2):113-9.

8. Sokos D, Everts V, de Vries TJ. Role of periodontal ligament fibroblasts in osteoclastogenesis: a review. J Periodontal Res. 2015;50(2):152-9.

9. Kanzaki H, Chiba M, Shimizu Y, Mitani H. Periodontal ligament cells under mechanical stress induce osteoclastogenesis by receptor activator of nuclear factor kappaB ligand up-regulation via prostaglandin E2 synthesis. J Bone Miner Res. 2002:17(2):210-20.

10. Nishijima Y, Yamaguchi M, Kojima T, Aihara N, Nakajima R, Kasai K. Levels of RANKL and OPG in gingival crevicular fluid during orthodontic tooth movement and effect of compression force on releases from periodontal ligament cells in vitro. Orthod Craniofac Res. 2006;9(2):63-70.

11. Yamaguchi M, Aihara N, Kojima T, Kasai K. RANKL increase in compressed periodontal ligament cells from root resorption. J Dent Res. 2006;85(8):751-6.

12. Tan L, Ren $Y$, Wang J, Jiang $L$, Cheng $H$, Sandham A, Zhao Z. Osteoprotegerin and ligand of receptor activator of nuclear factor kappaB expression in ovariectomized rats during tooth movement. Angle Orthod. 2009;79(2):292-8.

13. Garlet TP, Coelho U, Silva JS, Garlet GP. Cytokine expression pattern in compression and tension sides of the periodontal ligament during orthodontic tooth movement in humans. Eur J Oral Sci. 2007;115(5): 355-62.

14. Li S, Zhang H, Li S, Yang Y, Huo B, Zhang D. Connexin 43 and ERK regulate tension-induced signal transduction in human periodontal ligament fibroblasts. J Orthop Res. 2015;33(7):1008-14.

15. Kanzaki H, Chiba M, Sato A, Miyagawa A, Arai K, Nukatsuka S, Mitani H. Cyclical tensile force on periodontal ligament cells inhibits osteoclastogenesis through OPG induction. J Dent Res. 2006:85(5):457-62.

16. Jacobs C, Grimm S, Ziebart T, Walter C, Wehrbein H. Osteogenic differentiation of periodontal fibroblasts is dependent on the strength of mechanical strain. Arch Oral Biol. 2013;58(7):896-904. 
17. Tsuji K, Uno K, Zhang GX, Tamura M. Periodontal ligament cells under intermittent tensile stress regulate mRNA expression of osteoprotegerin and tissue inhibitor of matrix metalloprotease-1 and -2. J Bone Miner Metab. 2004;22(2):94-103.

18. Marin T, Gongol B, Chen Z, Woo B, Subramaniam S, Chien S, Shyy JY. Mechanosensitive microRNAs-role in endothelial responses to shear stress and redox state. Free Radic Biol Med. 2013;64:61-8.

19. Hua W, Zhang M, Wang Y, Yu L, Zhao T, Qiu X, Wang L. Mechanical stretch regulates microRNA expression profile via NF-kappaB activation in C2C12 myoblasts. Mol Med Rep. 2016;14(6):5084-92.

20. Yuan Y, Zhang L, Tong X, Zhang M, Zhao Y, Guo J, Lei L, Chen X, Tickner J, $\mathrm{Xu}$ J, et al. Mechanical stress regulates bone metabolism through MicroRNAs. J Cell Physiol. 2017;232(6):1239-45.

21. Yasuda H, Shima N, Nakagawa N, Yamaguchi K, Kinosaki M, Mochizuki S, Tomoyasu A, Yano K, Goto M, Murakami A, et al. Osteoclast differentiation factor is a ligand for osteoprotegerin/osteoclastogenesisinhibitory factor and is identical to TRANCE/RANKL. Proc Natl Acad Sci U S A. 1998;95(7):3597-602.

22. Yang $Y$, Yang $Y$, Li X, Cui L, Fu M, Rabie AB, Zhang D. Functional analysis of core binding factor $\mathrm{a} 1$ and its relationship with related genes expressed by human periodontal ligament cells exposed to mechanical stress. Eur J Orthod. 2010;32(6):698-705.

23. Kikuta J, Yamaguchi M, Shimizu M, Yoshino T, Kasai K. Notch signaling induces root resorption via RANKL and IL-6 from hPDL cells. J Dent Res. 2015;94(1):140-7.

24. Li Y, Zheng W, Liu JS, Wang J, Yang P, Li ML, Zhao ZH. Expression of osteoclastogenesis inducers in a tissue model of periodontal ligament under compression. J Dent Res. 2011;90(1):115-20.

25. Chen N, Sui BD, Hu CH, Cao J, Zheng CX, Hou R, Yang ZK, Zhao P, Chen Q, Yang QJ, et al. microRNA-21 contributes to orthodontic tooth movement. J Dent Res. 2016;95(12):1425-33.

26. Chang $M$, Lin $H$, Luo $M$, Wang J, Han G. Integrated miRNA and mRNA expression profiling of tension force-induced bone formation in periodontal ligament cells. In Vitro Cell Dev Biol Anim. 2015:51(8):797-807.

27. Nilforoushan D, Manolson MF. Expression of nitric oxide synthases in orthodontic tooth movement. Angle Orthod. 2009;79(3):502-8.

28. Tang N, Zhao Z, Zhang L, Yu Q, Li J, Xu Z, Li X. Up-regulated osteogenic transcription factors during early response of human periodontal ligament stem cells to cyclic tensile strain. Arch Med Sci. 2012;8(3):422-30.

29. Liu L, Liu M, Li R, Liu H, Du L, Chen H, Zhang Y, Zhang S, Liu D. MicroRNA503-5p inhibits stretch-induced osteogenic differentiation and bone formation. Cell Biol Int. 2017;41(2):112-23.

30. Chen Y, Mohammed A, Oubaidin M, Evans CA, Zhou X, Luan X, Diekwisch TG, Atsawasuwan P. Cyclic stretch and compression forces alter microRNA29 expression of human periodontal ligament cells. Gene. 2015;566(1):13-7.

31. Pitari MR, Rossi M, Amodio N, Botta C, Morelli E, Federico C, Gulla A, Caracciolo D, Di Martino MT, Arbitrio M, et al. Inhibition of miR-21 restores RANKL/OPG ratio in multiple myeloma-derived bone marrow stromal cells and impairs the resorbing activity of mature osteoclasts. Oncotarget. 2015;6(29):27343-58.

32. Hu CH, Sui BD, Du FY, Shuai Y, Zheng CX, Zhao P, Yu XR, Jin Y. miR-21 deficiency inhibits osteoclast function and prevents bone loss in mice. Sci Rep. 2017;7:43191.

33. Jia J, Zhou H, Zeng X, Feng S. Estrogen stimulates osteoprotegerin expression via the suppression of miR-145 expression in MG-63 cells. Mol Med Rep. 2017;15(4):1539-46

34. Chen P, Wei D, Xie B, Ni J, Xuan D, Zhang J. Effect and possible mechanism of network between microRNAs and RUNX2 gene on human dental follicle cells. J Cell Biochem. 2014;115(2):340-8.

35. Choi SW, Lee SU, Kim EH, Park SJ, Choi I, Kim TD, Kim SH. Osteoporotic bone of miR-150-deficient mice: possibly due to low serum OPGmediated osteoclast activation. Bone Rep. 2015;3:5-10.

36. Hong L, Sharp T, Khorsand B, Fischer C, Eliason S, Salem A, Akkouch A, Brogden K, Amendt BA. MicroRNA-200c represses IL-6, IL-8, and CCL-5 expression and enhances osteogenic differentiation. PLoS One. 2016;11(8):e0160915.

37. Rubin J, Rubin C, Jacobs CR. Molecular pathways mediating mechanical signaling in bone. Gene. 2006;367:1-16.

38. Persson H, Kvist A, Rego N, Staaf J, Vallon-Christersson J, Luts L, Loman N, Jonsson $G$, Naya $H$, Hoglund $M$, et al. Identification of new microRNAs in paired normal and tumor breast tissue suggests a dual role for the ERBB2/ Her2 gene. Cancer Res. 2011;71(1):78-86.
39. Kitagawa M, Tahara H, Kitagawa S, Oka H, Kudo Y, Sato S, Ogawa I, Miyaichi M, Takata T. Characterization of established cementoblast-like cell lines from human cementum-lining cells in vitro and in vivo. Bone. 2006;39(5):1035-42.

40. Wong N, Wang X. miRDB: an online resource for microRNA target prediction and functional annotations. Nucleic Acids Res. 2015;43(Database issue):D146-52.

41. Betel D, Wilson M, Gabow A, Marks DS, Sander C. The microRNA.org resource: targets and expression. Nucleic Acids Res. 2008;36(Database issue): D149-53.

\section{Ready to submit your research? Choose BMC and benefit from:}

- fast, convenient online submission

- thorough peer review by experienced researchers in your field

- rapid publication on acceptance

- support for research data, including large and complex data types

- gold Open Access which fosters wider collaboration and increased citations

- maximum visibility for your research: over $100 \mathrm{M}$ website views per year

At BMC, research is always in progress.

Learn more biomedcentral.com/submissions 